Ann. Funct. Anal. 6 (2015), no. 4, 191-205

http://doi.org/10.15352/afa/06-4-191

ISSN: 2008-8752 (electronic)

http://projecteuclid.org/afa

\title{
COMMUTATORS OF CONVOLUTION TYPE OPERATORS ON SOME BANACH FUNCTION SPACES
}

\author{
ALEXEI YU. KARLOVICH \\ Communicated by I. Spitkovsky
}

\begin{abstract}
We study the boundedness of Fourier convolution operators $W^{0}(b)$ and the compactness of commutators of $W^{0}(b)$ with multiplication operators $a I$ on some Banach function spaces $X(\mathbb{R})$ for certain classes of piecewise quasicontinuous functions $a \in P Q C$ and piecewise slowly oscillating Fourier multipliers $b \in P S O_{X, 1}^{\diamond}$. We suppose that $X(\mathbb{R})$ is a separable rearrangement-invariant space with nontrivial Boyd indices or a reflexive variable Lebesgue space, in which the Hardy-Littlewood maximal operator is bounded. Our results complement those of Isaac De La Cruz-Rodríguez, Yuri Karlovich, and Iván Loreto Hernández obtained for Lebesgue spaces with Muckenhoupt weights.
\end{abstract}

\section{INTRODUCTION AND PRELIMINARIES}

Let $F: L^{2}(\mathbb{R}) \rightarrow L^{2}(\mathbb{R})$ denote the Fourier transform

$$
(F f)(x):=\widehat{f}(x):=\int_{\mathbb{R}} f(t) e^{i t x} d t, \quad x \in \mathbb{R},
$$

and let $F^{-1}: L^{2}(\mathbb{R}) \rightarrow L^{2}(\mathbb{R})$ be the inverse of $F$,

$$
\left(F^{-1} g\right)(t)=\frac{1}{2 \pi} \int_{\mathbb{R}} g(x) e^{-i t x} d x, \quad t \in \mathbb{R} .
$$

It is well known that the Fourier convolution operator $W^{0}(a):=F^{-1} a F$ is bounded on the space $L^{2}(\mathbb{R})$ for every $a \in L^{\infty}(\mathbb{R})$.

We will study Fourier convolution operators on (weighted) Banach function spaces. Let $\mathbb{R}_{+}:=(0, \infty)$ and $\mathbb{S} \in\left\{\mathbb{R}_{+}, \mathbb{R}\right\}$. The set of all Lebesgue measurable

Date: Received: Feb. 23, 2015; Accepted: May 12, 2015.

2010 Mathematics Subject Classification. Primary 47B47; Secondary 42A45, 46 E30.

Key words and phrases. Fourier convolution operator, commutator, piecewise quasicontinuous function, piecewise slowly oscillating multiplier, Banach function space, rearrangementinvariant space, variable Lebesgue space. 
complex-valued functions on $\mathbb{S}$ is denoted by $\mathfrak{M}(\mathbb{S})$. Let $\mathfrak{M}^{+}(\mathbb{S})$ be the subset of functions in $\mathfrak{M}(\mathbb{S})$ whose values lie in $[0, \infty]$. The Lebesgue measure of a measurable set $E \subset \mathbb{S}$ is denoted by $|E|$ and its characteristic function is denoted by $\chi_{E}$. Following [1, Chap. 1, Definition 1.1], a mapping $\rho: \mathfrak{M}^{+}(\mathbb{S}) \rightarrow[0, \infty]$ is called a Banach function norm if, for all functions $f, g, f_{n}(n \in \mathbb{N})$ in $\mathfrak{M}^{+}(\mathbb{S})$, for all constants $a \geq 0$, and for all measurable subsets $E$ of $\mathbb{S}$, the following properties hold:

(A1) $\quad \rho(f)=0 \Leftrightarrow f=0$ a.e., $\quad \rho(a f)=a \rho(f), \quad \rho(f+g) \leq \rho(f)+\rho(g)$,

(A2) $0 \leq g \leq f$ a.e. $\Rightarrow \rho(g) \leq \rho(f) \quad$ (the lattice property),

(A3) $0 \leq f_{n} \uparrow f$ a.e. $\Rightarrow \rho\left(f_{n}\right) \uparrow \rho(f) \quad$ (the Fatou property),

(A4) $|E|<\infty \Rightarrow \rho\left(\chi_{E}\right)<\infty$,

$$
|E|<\infty \Rightarrow \int_{E} f(x) d x \leq C_{E} \rho(f)
$$

with $C_{E} \in(0, \infty)$ which may depend on $E$ and $\rho$ but is independent of $f$. When functions differing only on a set of measure zero are identified, the set $X(\mathbb{S})$ of all functions $f \in \mathfrak{M}(\mathbb{S})$ for which $\rho(|f|)<\infty$ is called a Banach function space. For each $f \in X(\mathbb{S})$, the norm of $f$ is defined by

$$
\|f\|_{X(\mathbb{S})}:=\rho(|f|) .
$$

Under the natural linear space operations and under this norm, the set $X(\mathbb{S})$ becomes a Banach space (see [1, Chap. 1, Theorems 1.4 and 1.6]). If $\rho$ is a Banach function norm, its associate norm $\rho^{\prime}$ is defined on $\mathfrak{M}^{+}(\mathbb{S})$ by

$$
\rho^{\prime}(g):=\sup \left\{\int_{\mathbb{S}} f(x) g(x) d x: f \in \mathfrak{M}^{+}(\mathbb{S}), \rho(f) \leq 1\right\}, \quad g \in \mathfrak{M}^{+}(\mathbb{S}) .
$$

It is a Banach function norm itself [1, Chap. 1, Theorem 2.2]. The Banach function space $X^{\prime}(\mathbb{R})$ determined by the Banach function norm $\rho^{\prime}$ is called the associate space (Köthe dual) of $X(\mathbb{S})$. The associate space $X^{\prime}(\mathbb{S})$ is a subspace of the (Banach) dual space $[X(\mathbb{S})]^{*}$.

Let $\mathfrak{M}_{0}(\mathbb{S})$ and $\mathfrak{M}_{0}^{+}(\mathbb{S})$ be the classes of a.e. finite functions in $\mathfrak{M}(\mathbb{S})$ and $\mathfrak{M}^{+}(\mathbb{S})$, respectively. The distribution function $\mu_{f}$ of $f \in \mathfrak{M}_{0}(\mathbb{S})$ is given by

$$
\mu_{f}(\lambda):=|\{x \in \mathbb{S}:|f(x)|>\lambda\}|, \quad \lambda \geq 0 .
$$

Two functions $f, g \in \mathfrak{M}_{0}(\mathbb{S})$ are said to be equimeasurable if $\mu_{f}(\lambda)=\mu_{g}(\lambda)$ for all $\lambda \geq 0$. The non-increasing rearrangement of $f \in \mathfrak{M}_{0}(\mathbb{S})$ is the function defined by

$$
f^{*}(t):=\inf \left\{\lambda: \mu_{f}(\lambda) \leq t\right\}, \quad t \geq 0 .
$$

We here use the standard convention that inf $\emptyset=+\infty$. A Banach function norm $\rho: \mathfrak{M}^{+}(\mathbb{S}) \rightarrow[0, \infty]$ is called rearrangement-invariant if for every pair of equimeasurable functions $f, g \in \mathfrak{M}_{0}^{+}(\mathbb{S})$ the equality $\rho(f)=\rho(g)$ holds. In that case, the Banach function space $X(\mathbb{S})$ generated by $\rho$ is said to be a rearrangementinvariant Banach function space (or simply rearrangement-invariant space).

Lebesgue, Orlicz, and Lorentz spaces are classical examples of rearrangementinvariant Banach function spaces (see, e.g., [1] and the references therein). By 
[1, Chap. 2, Proposition 4.2], if a Banach function space $X(\mathbb{S})$ is rearrangementinvariant, then its associate space $X^{\prime}(\mathbb{S})$ is rearrangement-invariant, too.

Now we give an example of a Banach function space which is not rearrangementinvariant. Let $p: \mathbb{R} \rightarrow[1, \infty]$ be a measurable a.e. finite function. By $L^{p(\cdot)}(\mathbb{R})$ we denote the set of all complex-valued functions $f$ on $\mathbb{R}$ such that

$$
I_{p(\cdot)}(f / \lambda):=\int_{\mathbb{R}}|f(x) / \lambda|^{p(x)} d x<\infty
$$

for some $\lambda>0$. This set becomes a Banach function space when equipped with the norm

$$
\|f\|_{L^{p(\cdot)(\mathbb{R})}}:=\inf \left\{\lambda>0: I_{p(\cdot)}(f / \lambda) \leq 1\right\} .
$$

It is easy to see that if $p$ is constant, then $L^{p(\cdot)}(\mathbb{R})$ is nothing but the standard Lebesgue space $L^{p}(\mathbb{R})$. The space $L^{p(\cdot)}(\mathbb{R})$ is referred to as a variable Lebesgue space, it is a Banach function space [7, Section 2.10.3]. If $p: \mathbb{R} \rightarrow[1, \infty]$ is not constant, then $L^{p(\cdot)}(\mathbb{R})$ is not rearrangement-invariant [7, Example 3.14]. Put

$$
p_{-}:=\underset{x \in \mathbb{R}}{\operatorname{essinf}} p(x), \quad p_{+}:=\underset{x \in \mathbb{R}}{\operatorname{ess} \sup } p(x) .
$$

The space $L^{p(\cdot)}(\mathbb{R})$ is separable if and only if $p_{+}<\infty[7$, Theorem 2.78]. Finally, by $\left[7\right.$, Theorem 2.80, Corollary 2.81], the space $L^{p(\cdot)}(\mathbb{R})$ is reflexive if and only if

$$
1<p_{-}, \quad p_{+}<\infty .
$$

In the latter case, the associate space $\left(L^{p(\cdot)}(\mathbb{R})\right)^{\prime}$ is isomorphic to the space $L^{p^{\prime}(\cdot)}(\mathbb{R})$, where $1 / p(x)+1 / p^{\prime}(x)=1$ for a.e. $x \in \mathbb{R}$.

A function $w \in \mathfrak{M}^{+}(\mathbb{R})$ is referred to as a weight if $0<w(x)<\infty$ a.e. on $\mathbb{R}$. Define the weighted Banach function space $X(\mathbb{R}, w)$ as the set of all complexvalued measurable functions $f$ on $\mathbb{R}$ such that $f w \in \mathbb{R}$. This is a linear normed space when equipped with the norm

$$
\|f\|_{X(\mathbb{R}, w)}:=\|f w\|_{X(\mathbb{R})} .
$$

We say that $f \in X_{\text {loc }}(\mathbb{R})$ if $f \chi_{E} \in X(\mathbb{R})$ for any measurable set $E \subset \mathbb{R}$ of finite measure. If $w \in X_{\text {loc }}(\mathbb{R})$ and $1 / w \in X_{\text {loc }}^{\prime}(\mathbb{R})$, then $X(\mathbb{R}, w)$ is a Banach function space itself and $X^{\prime}\left(\mathbb{R}, w^{-1}\right)$ is its associate space [15, Lemma 2.4]. If, in addition, $X(\mathbb{R})$ is separable, then the set of all bounded compactly supported functions is dense in $X(\mathbb{R}, w)[15$, Lemma $2.12(\mathrm{a})]$, which implies that $L^{2}(\mathbb{R}) \cap X(\mathbb{R}, w)$ is dense in $X(\mathbb{R}, w)$. If $X(\mathbb{R})$ is a Banach function space and $w: \mathbb{R} \rightarrow[0, \infty]$ is a weight such that the Cauchy singular integral operator $S_{\mathbb{R}}$ given by

$$
\left(S_{\mathbb{R}} f\right)(x):=\lim _{\varepsilon \rightarrow 0} \frac{1}{\pi i} \int_{\mathbb{R} \backslash(x-\varepsilon, x+\varepsilon)} \frac{f(t)}{t-x} d t, \quad x \in \mathbb{R},
$$

is bounded on the (normed) space $X(\mathbb{R}, w)$, then, by [15, Theorem 3.9], $X(\mathbb{R}, w)$ is a Banach function space and

$$
\sup _{I} \frac{1}{|I|}\left\|w \chi_{I}\right\|_{X(\mathbb{R})}\left\|w^{-1} \chi_{I}\right\|_{X^{\prime}(\mathbb{R})}<\infty
$$

where the supremum is taken over all bounded intervals $I \subset \mathbb{R}$. In the latter case we write $w \in A_{X}(\mathbb{R})$. We will use the following convention $A_{p}(\mathbb{R}):=A_{L^{p}}(\mathbb{R})$ for 
$1<p<\infty$. The class $A_{p}(\mathbb{R})$ is usually called the Muckenhoupt class because Muckenhoupt proved in [20, Theorem 2] that $w \in A_{p}(\mathbb{R})$ if and only if the HardyLittlewood maximal operator defined for $f \in L_{\mathrm{loc}}^{1}(\mathbb{R})$ by

$$
(M f)(x):=\sup _{I \ni x} \frac{1}{|I|} \int_{I}|f(y)| d y,
$$

where the supremum is taken over all finite intervals $I \subset \mathbb{R}$ containing $x$, is bounded on $L^{p}(\mathbb{R}, w), 1<p<\infty$. Later Hunt, Muckenhoupt, and Wheeden [11, Theorem 9], proved that $S_{\mathbb{R}}$ is bounded on the weighted Lebesgue space $L^{p}(\mathbb{R}, w)$, $1<p<\infty$, if and only if $w \in A_{p}(\mathbb{R})$.

Let $X(\mathbb{R})$ be a separable Banach function space and $w: \mathbb{R} \rightarrow[0, \infty]$ be a weight. A function $a \in L^{\infty}(\mathbb{R})$ is called a Fourier multiplier on $X(\mathbb{R}, w)$ if the map $f \mapsto F^{-1} a F$ maps $L^{2}(\mathbb{R}) \cap X(\mathbb{R}, w)$ into $X(\mathbb{R}, w)$ and extends to a bounded linear operator on $X(\mathbb{R}, w)$ denoted by $W^{0}(a)$. The set of all Fourier multipliers on $X(\mathbb{R}, w)$ is denoted by $\mathcal{M}_{X, w}(\mathbb{R})$. It is clear that $\mathcal{M}_{X, w}(\mathbb{R})$ is a unital normed algebra under pointwise operations and the norm

$$
\|a\|_{\mathcal{M}_{X, w}(\mathbb{R})}:=\left\|W^{0}(a)\right\|_{\mathcal{L}(X(\mathbb{R}, w))} .
$$

Here and in what follows $\mathcal{L}(X(\mathbb{R}, w))$ denotes the Banach algebra of all bounded linear operators on $X(\mathbb{R}, w)$. If the operator $S_{\mathbb{R}}$ is bounded on $X(\mathbb{R}, w)$, then

$$
\|a\|_{L^{\infty}(\mathbb{R})} \leq c_{X, w}\|a\|_{\mathcal{M}_{X, w}(\mathbb{R})}
$$

for all $a \in \mathcal{M}_{X, w}(\mathbb{R})$, where $c_{X, w}$ is a positive constant depending only on $X(\mathbb{R})$ and $w$. Moreover, $\mathcal{M}_{X, w}(\mathbb{R})$ is a Banach algebra [13, Theorem 1, Corollary 1].

The aim of this paper is to study the boundedness of convolution operators $W^{0}(b)$ on weighted Banach function spaces $X(\mathbb{R}, w)$ and the compactness of commutators of multiplication operators $a I$ and convolution operators $W^{0}(b)$ on separable rearrangement-invariant Banach function spaces $X(\mathbb{R})$ and on reflexive variable Lebesgue spaces $L^{p(\cdot)}(\mathbb{R})$. Our results complement a series of results obtained for standard Lebesgue spaces with weights in [10, Lemmas 7.1-7.4] for piecewise continuous functions $a, b$; in [17, Theorem 4.6] for piecewise slowly oscillating functions $a, b$; and in [8, Theorem 4.4] for wider classes of piecewise quasicontinuous functions $a, b$. Note that as soon as results on the compactness of commutators $a W^{0}(b)-W^{0}(b) a I$ are available, one can employ the Allan-Douglas local principle (see, e.g., [2, Theorem 8.2]) and the two projections theorem (see, e.g., [2, Theorem 8.7]) and develop a theory of Fredholmness (that is, invertibility modulo the ideal of compact operators) for algebras generated by $a I$ and $W^{0}(b)$ (see, e.g., [16] for weighted Lebesgue spaces). We plan to address these questions in a forthcoming paper.

The paper is organized as follows. In Section 2 we formulate our results on the boundedness of convolution operators $W^{0}(a)$ with symbols in the algebra $V(\mathbb{R})$ of functions of bounded total variation and in the algebras $\mathrm{SO}_{\lambda}^{3}$ of three times continuously differentiable functions that slowly oscillate at $\lambda \in \mathbb{R} \cup\{\infty\}$. Further, we define the algebras of functions $C(\overline{\mathbb{R}}), P C, S O^{\diamond}, Q C, P S O^{\diamond}$, all being subalgebras of the algebra $P Q C$ of the piecewise quasicontinuous functions, and the corresponding algebras of Fourier multipliers $C_{X, w}(\overline{\mathbb{R}}), P C_{X, w}, S O_{X, w}^{\diamond}, P S O_{X, w}^{\diamond}$. 
The latter definitions rely on the above mentioned boundedness results. Finally, we formulate the result on the compactness of the commutator $a W^{0}(b)-W^{0}(b) a I$. In Sections 3 and 4 we prove the boundedness results for $W^{0}(a)$ and in Section 5 we give a proof of the compactness result for the commutator $a W^{0}(b)-W^{0}(b) a I$.

\section{MAin RESUlts}

2.1. Convolution operators with symbols in the algebra $V(\mathbb{R})$. Let $a$ be a complex-valued function of bounded total variation $V(a)$ on $\mathbb{R}$ where

$$
V(a):=\sup \left\{\sum_{k=1}^{n}\left|a\left(x_{k}\right)-a\left(x_{k-1}\right)\right|:-\infty<x_{0}<x_{1}<\cdots<x_{n}<+\infty, n \in \mathbb{N}\right\} .
$$

The set $V(\mathbb{R})$ of all functions of bounded total variation on $\mathbb{R}$ is a unital nonseparable Banach algebra with the norm

$$
\|a\|_{V}:=\|a\|_{L^{\infty}(\mathbb{R})}+V(a) .
$$

It is well known that the algebra $V(\mathbb{R})$ is continuously embedded into $\mathcal{M}_{L^{p}, w}(\mathbb{R})$ in the case of standard Lebesgue spaces $L^{p}(\mathbb{R}), 1<p<\infty$, and Muckenhoupt weights $w \in A_{p}(\mathbb{R})$. Moreover, the embedding constant is equal to $\left\|S_{\mathbb{R}}\right\|_{\mathcal{L}\left(L^{p}(\mathbb{R}, w)\right)}$ (see [10, Theorem 2.11] for $w=1$ and [3, Theorem 17.1] for $w \in A_{p}(\mathbb{R})$ ). Now we formulate the generalization of this result to weighted Banach function spaces.

Theorem 2.1. Let $w: \mathbb{R} \rightarrow[0, \infty]$ be a weight and $X(\mathbb{R})$ be a separable Banach function space such that $w \in X_{\mathrm{loc}}(\mathbb{R})$ and $1 / w \in X_{\mathrm{loc}}^{\prime}(\mathbb{R})$. Suppose the HardyLittlewood maximal operator is bounded on the spaces $X(\mathbb{R}, w)$ and $X^{\prime}\left(\mathbb{R}, w^{-1}\right)$. If a has a finite total variation $V(a)$, then the convolution operator $W^{0}(a)$ is bounded on the space $X(\mathbb{R}, w)$ and

$$
\left\|W^{0}(a)\right\|_{\mathcal{L}(X(\mathbb{R}, w))} \leq c_{X, w}\|a\|_{V}
$$

where $c_{X, w}$ is a positive constant depending only on $X(\mathbb{R})$ and $w$.

This result follows from [15, Lemma 2.4(b)] and [12, Theorem 4.3].

If $X(\mathbb{R})$ is a rearrangement-invariant space or a variable Lebesgue space, conditions for the boundedness of the Hardy-Littlewood maximal operator on $X(\mathbb{R}, w)$ are known, this observation allows us to simplify the formulation of the above result.

Let $X(\mathbb{R})$ be a rearrangement-invariant Banach function space generated by a rearrangement-invariant Banach function norm $\rho$. In this case, the Luxemburg representation theorem [1, Chap. 2, Theorem 4.10] provides a unique rearrangement-invariant Banach function norm $\bar{\rho}$ over $\mathbb{R}_{+}$equipped with the Lebesgue measure, defined by

$$
\bar{\rho}(h):=\sup \left\{\int_{\mathbb{R}_{+}} g^{*}(t) h^{*}(t) d t: \rho^{\prime}(g) \leq 1\right\},
$$

and such that $\rho(f)=\bar{\rho}\left(f^{*}\right)$ for all $f \in \mathfrak{M}_{0}^{+}(\mathbb{R})$. The rearrangement-invariant Banach function space generated by $\bar{\rho}$ is denoted by $\bar{X}\left(\mathbb{R}_{+}\right)$.

For each $t>0$, let $E_{t}$ denote the dilation operator defined on $\mathfrak{M}\left(\mathbb{R}_{+}\right)$by

$$
\left(E_{t} f\right)(s)=f(s t), \quad 0<s<\infty .
$$


With $X(\mathbb{R})$ and $\bar{X}\left(\mathbb{R}_{+}\right)$as above, let $h_{X}(t)$ denote the operator norm of $E_{1 / t}$ as an operator on $\bar{X}\left(\mathbb{R}_{+}\right)$. By [1, Chap. 3, Proposition 5.11], for each $t>0$, the operator $E_{t}$ is bounded on $\bar{X}\left(\mathbb{R}_{+}\right)$and the function $h_{X}$ is increasing and submultiplicative on $(0, \infty)$. The Boyd indices of $X(\mathbb{R})$ are the numbers $\alpha_{X}$ and $\beta_{X}$ defined by

$$
\alpha_{X}:=\sup _{t \in(0,1)} \frac{\log h_{X}(t)}{\log t}, \quad \beta_{X}:=\inf _{t \in(1, \infty)} \frac{\log h_{X}(t)}{\log t} .
$$

By [1, Chap. 3, Proposition 5.13], $0 \leq \alpha_{X} \leq \beta_{X} \leq 1$. The Boyd indices are said to be nontrivial if $\alpha_{X}, \beta_{X} \in(0,1)$. The Boyd indices of the Lebesgue space $L^{p}(\mathbb{R}), 1 \leq p \leq \infty$, are both equal to $1 / p$. Note that the Boyd indices of a rearrangement-invariant space may be different [1, Chap. 3, Exercises 6, 13].

Corollary 2.2. Let $X(\mathbb{R})$ be a separable rearrangement-invariant space with nontrivial Boyd indices $\alpha_{X}, \beta_{X}$ and $w \in A_{1 / \alpha_{X}}(\mathbb{R}) \cap A_{1 / \beta_{X}}(\mathbb{R})$. If a has a finite total variation $V(a)$, then the convolution operator $W^{0}(a)$ is bounded on the weighted rearrangement-invariant space $X(\mathbb{R}, w)$ and

$$
\left\|W^{0}(a)\right\|_{\mathcal{L}(X(\mathbb{R}, w))} \leq c_{X, w}\|a\|_{V}
$$

where $c_{X, w}$ is a positive constant depending only on $X(\mathbb{R})$ and $w$.

Corollary 2.2 will be proved in Section 4.3.

By $\mathcal{B}_{M}(\mathbb{R})$ denote the set of all measurable functions $p: \mathbb{R} \rightarrow[1, \infty]$ such that (1.1) holds and the Hardy-Littlewood maximal operator is bounded on the variable Lebesgue space $L^{p(\cdot)}(\mathbb{R})$. To provide a simple sufficient conditions guaranteeing that $p \in \mathcal{B}_{M}(\mathbb{R})$, we need the following definition. Given a function $r: \mathbb{R} \rightarrow \mathbb{R}$, one says that $r$ is locally log-Hölder continuous if there exists a constant $C_{0}>0$ such that

$$
|r(x)-r(y)| \leq \frac{C_{0}}{-\log |x-y|}
$$

for all $x, y \in \mathbb{R}$ such that $|x-y|<1 / 2$. One says that $r: \mathbb{R} \rightarrow \mathbb{R}$ is log-Hölder continuous at infinity if there exist constants $C_{\infty}$ and $r_{\infty}$ such that for all $x \in \mathbb{R}$,

$$
\left|r(x)-r_{\infty}\right| \leq \frac{C_{\infty}}{\log (e+|x|)} .
$$

The class of functions $r: \mathbb{R} \rightarrow \mathbb{R}$ that are simultaneously locally log-Hölder continuous and log-Hölder continuous at infinity is denoted by $L H(\mathbb{R})$. From $[7$, Proposition 2.3 and Theorem 3.16] we deduce that if $p \in L H(\mathbb{R})$ satisfies (1.1), then $p \in \mathcal{B}_{M}(\mathbb{R})$.

Corollary 2.3. Let $p \in L H(\mathbb{R})$ satisfy $(1.1)$ and $w \in A_{L^{p(\cdot)}}(\mathbb{R})$. If a has a finite total variation $V(a)$, then the convolution operator $W^{0}(a)$ is bounded on the weighted variable Lebesgue space $L^{p(\cdot)}(\mathbb{R}, w)$ and

$$
\left\|W^{0}(a)\right\|_{\mathcal{L}\left(L^{p(\cdot)}(\mathbb{R}, w)\right)} \leq c_{p(\cdot), w}\|a\|_{V}
$$

where $c_{p(\cdot), w}$ is a positive constant depending only on the variable exponent $p$ and the weight $w$. 
Corollary 2.3 will be proved in Section 4.4.

Although $p \in L H(\mathbb{R})$ provides a nice sufficient condition for the boundedness of the Hardy-Littlewood maximal operator on the variable Lebesgue space $L^{p(\cdot)}(\mathbb{R})$, it is not necessary. Notice that all functions in $L H(\mathbb{R})$ are continuous and have limits at infinity. An example of an exponent $p \in \mathcal{B}_{M}(\mathbb{R})$, which does not have limits at zero and infinity, and thus $p \notin L H(\mathbb{R})$, is given in [7, Example 4.68], [9, Section 5.1]. We refer to the recent monographs $[7,9]$ for further discussions concerning the fascinating and still mysterious class $\mathcal{B}_{M}(\mathbb{R})$.

Corollary 2.4. Let $p \in \mathcal{B}_{M}$. If a has a finite total variation $V(a)$, then the convolution operator $W^{0}(a)$ is bounded on the variable Lebesgue space $L^{p(\cdot)}(\mathbb{R})$ and

$$
\left\|W^{0}(a)\right\|_{\mathcal{L}\left(L^{p(\cdot)}(\mathbb{R})\right)} \leq c_{p(\cdot)}\|a\|_{V}
$$

where $c_{p(\cdot)}$ is a positive constant depending only on the variable exponent $p$.

Corollary 2.4 will be proved in Section 4.5.

2.2. Convolution operators with symbols in the algebra $S O_{\lambda}^{3}$. Consider the one point compactification of the real line $\dot{\mathbb{R}}:=\mathbb{R} \cup\{\infty\}$. For $\lambda \in \dot{\mathbb{R}}$, put

$$
C_{b}(\dot{\mathbb{R}} \backslash\{\lambda\}):=C(\dot{\mathbb{R}} \backslash\{\lambda\}) \cap L^{\infty}(\mathbb{R}) .
$$

For a bounded measurable function $f: \mathbb{R} \rightarrow \mathbb{C}$ and a set $I \subset \mathbb{R}$, let

$$
\operatorname{osc}(f, I):=\underset{x, y \in I}{\operatorname{ess} \sup }|f(x)-f(y)| \text {. }
$$

Following [17, Section 2.1], for $\lambda \in \dot{\mathbb{R}}$ consider the $C^{*}$-algebras $S O_{\lambda}$ of slowly oscillating functions at $\lambda$ defined by

$$
\begin{aligned}
S O_{\infty} & :=\left\{f \in C_{b}(\dot{\mathbb{R}} \backslash\{\infty\}): \lim _{x \rightarrow+\infty} \operatorname{osc}(f,[-x,-x / 2] \cup[x / 2, x])=0\right\}, \\
S O_{\lambda} & :=\left\{f \in C_{b}(\dot{\mathbb{R}} \backslash\{\lambda\}): \lim _{x \rightarrow+0} \operatorname{osc}(f, \lambda+[-x,-x / 2] \cup[x / 2, x])=0\right\}(\lambda \in \mathbb{R}) .
\end{aligned}
$$

Consider the differential operator $D_{\lambda}$ defined by

$$
\left(D_{\lambda} a\right)(x):=\left\{\begin{array}{ll}
(x-\lambda) a^{\prime}(x) & \text { for } \lambda \in \mathbb{R}, \\
x a^{\prime}(x) & \text { for } \lambda=\infty
\end{array} \quad(x \in \mathbb{R}) .\right.
$$

Following [17, Section 2.3], put

$$
S O_{\lambda}^{3}:=\left\{a \in S O_{\lambda} \cap C^{3}(\mathbb{R} \backslash\{\lambda\}): \lim _{x \rightarrow \lambda}\left(D_{\lambda}^{j} a\right)(x)=0, j=1,2,3\right\},
$$

where $D_{\lambda}^{0} f:=f$ and $D_{\lambda}^{j} f:=D_{\lambda}\left(D_{\lambda}^{j-1} f\right)$ for $j \in \mathbb{N}$. It is easy to see that $S O_{\lambda}^{3}$ is a commutative Banach algebra under the pointwise operations and the norm

$$
\|a\|_{S O_{\lambda}^{3}}:=\sum_{j=0}^{3} \frac{1}{j !}\left\|D_{\lambda}^{j} a\right\|_{L^{\infty}(\mathbb{R})} .
$$


Theorem 2.5. Let $X(\mathbb{R})$ be a separable Banach function space and $w: \mathbb{R} \rightarrow$ $[0, \infty]$ be a weight such that $w \in X_{\mathrm{loc}}(\mathbb{R})$ and $1 / w \in X_{\mathrm{loc}}^{\prime}(\mathbb{R})$. Suppose the HardyLittlewood maximal operator $M$ is bounded on $X(\mathbb{R}, w)$ and on $X^{\prime}\left(\mathbb{R}, w^{-1}\right)$. If $\lambda \in \dot{\mathbb{R}}$ and $a \in S O_{\lambda}^{3}$, then the convolution operator $W^{0}(a)$ is bounded on the space $X(\mathbb{R}, w)$ and

$$
\left\|W^{0}(a)\right\|_{\mathcal{L}(X(\mathbb{R}, w))} \leq c_{X, w}\|a\|_{S O_{\lambda}^{3}}
$$

where $c_{X, w}$ is a positive constant depending only on $X(\mathbb{R})$ and $w$.

We will prove Theorem 2.5 in Section 4.1.

Corollary 2.6. Let $X(\mathbb{R})$ be a separable rearrangement-invariant space with nontrivial Boyd indices $\alpha_{X}, \beta_{X}$ and $w \in A_{1 / \alpha_{X}}(\mathbb{R}) \cap A_{1 / \beta_{X}}(\mathbb{R})$. If $\lambda \in \dot{\mathbb{R}}$ and $a \in S O_{\lambda}^{3}$, then the convolution operator $W^{0}(a)$ is bounded on the space $X(\mathbb{R}, w)$ and

$$
\left\|W^{0}(a)\right\|_{\mathcal{L}(X(\mathbb{R}, w))} \leq c_{X, w}\|a\|_{S O_{\lambda}^{3}}
$$

where $c_{X, w}$ is a positive constant depending only on $X(\mathbb{R})$ and $w$.

A proof of Corollary 2.6 will be given in Section 4.3.

Corollary 2.7. Let $p \in L H(\mathbb{R})$ satisfy (1.1) and $w \in A_{L^{p(\cdot)}}(\mathbb{R})$. If $\lambda \in \dot{\mathbb{R}}$ and $a \in S O_{\lambda}^{3}$, then the convolution operator $W^{0}(a)$ is bounded on the space $L^{p(\cdot)}(\mathbb{R}, w)$ and

$$
\left\|W^{0}(a)\right\|_{\mathcal{L}\left(L^{p(\cdot)(\mathbb{R}, w))}\right.} \leq c_{p(\cdot), w}\|a\|_{S O_{\lambda}^{3}},
$$

where $c_{p(\cdot), w}$ is a positive constant depending only on the variable exponent $p$ and the weight $w$.

Corollary 2.7 will be proved in Section 4.4 .

Corollary 2.8. Let $p \in \mathcal{B}_{M}$. If $\lambda \in \dot{\mathbb{R}}$ and $a \in S O_{\lambda}^{3}$, then the convolution operator $W^{0}(a)$ is bounded on the variable Lebesgue space $L^{p(\cdot)}(\mathbb{R})$ and

$$
\left\|W^{0}(a)\right\|_{\mathcal{L}\left(L^{p(\cdot)}(\mathbb{R})\right)} \leq c_{p(\cdot)}\|a\|_{S O_{\lambda}^{3}}
$$

where $c_{p(\cdot)}$ is a positive constant depending only on the variable exponent $p$.

We will present a proof Corollary 2.8 in Section 4.5.

2.3. Compactness of commutators. Let $C(\dot{\mathbb{R}})$ and $C(\overline{\mathbb{R}})$ denote the $C^{*}$-algebras of continuous functions on $\dot{\mathbb{R}}$ and $\overline{\mathbb{R}}:=[-\infty,+\infty]$, respectively, and $P C$ stand for the $C^{*}$-algebra of all functions $f: \dot{\mathbb{R}} \rightarrow \mathbb{C}$ that possess finite one-sided limits

$$
f(x \pm 0):=\lim _{t \rightarrow x \pm 0} a(t)
$$

at every point $x \in \dot{\mathbb{R}}$, where $a( \pm \infty)=a(\infty \mp 0)$. Note that if $V(a)<\infty$, then $a \in P C$. Following [17, Section 2.1], let $S O^{\diamond}$ be the smallest $C^{*}$-subalgebra of $L^{\infty}(\mathbb{R})$ that contains all the $C^{*}$-algebras $S O_{\lambda}$ with $\lambda \in \dot{\mathbb{R}}$. In particular, $S O^{\diamond}$ contains $C(\dot{\mathbb{R}})$.

Let $H^{\infty}$ (resp. $\overline{H^{\infty}}$ ) be the closed subalgebra of $L^{\infty}(\mathbb{R})$ that consists of all functions being non-tangential limits on $\mathbb{R}$ of all bounded analytic functions on 
the upper (resp. lower) half-plane. Following [21, 22] consider the $C^{*}$-algebra $Q C$ of quasicontinuous functions on $\dot{\mathbb{R}}$ defined by

$$
Q C:=\left(H^{\infty}+C(\dot{\mathbb{R}})\right) \cap\left(\overline{H^{\infty}}+C(\dot{\mathbb{R}})\right),
$$

and the $C^{*}$-algebra $P Q C$ of piecewise quasicontinuous functions, which is the smallest $C^{*}$-subalgebra of $L^{\infty}(\mathbb{R})$ that contains $P C$ and $Q C$. By [17, Theorem 4.2], $S O^{\diamond} \subset Q C$. According to [17, Section 2.4], $P S O^{\diamond}$ is a smallest $C^{*}$ subalgebra of $L^{\infty}(\mathbb{R})$ that contains $P C$ and $S O^{\diamond}$. Thus $P S O^{\diamond} \subset P Q C$.

For a Banach algebra $\mathfrak{A}$ and its subset $\mathfrak{S}$, let $\operatorname{alg}_{\mathfrak{A}} \mathfrak{S}$ denote the smallest Banach subalgebra of $\mathfrak{A}$ that contains the set $\mathfrak{S}$.

Let $w: \mathbb{R} \rightarrow[0, \infty]$ be a weight and $X(\mathbb{R})$ be a separable Banach function space such that $w \in X_{\text {loc }}(\mathbb{R})$ and $1 / w \in X_{\text {loc }}^{\prime}(\mathbb{R})$. Suppose the Hardy-Littlewood maximal operator is bounded on the spaces $X(\mathbb{R}, w)$ and $X^{\prime}\left(\mathbb{R}, w^{-1}\right)$. By $[15$, Theorem 3.8] and [13, Theorem 1, Corollary 1], $\mathcal{M}_{X, w}(\mathbb{R}) \subset L^{\infty}(\mathbb{R})$ and $\mathcal{M}_{X, w}(\mathbb{R})$ is a Banach algebra. From Theorems 2.1 and 2.5 we know that $V(\mathbb{R}) \subset \mathcal{M}_{X, w}(\mathbb{R})$ and $S O_{\lambda}^{3} \subset \mathcal{M}_{X, w}(\mathbb{R})$ for all $\lambda \in \dot{\mathbb{R}}$. By analogy with [10, Chap. I], [17, Section 2.4], [18, Section 3], put

$$
\begin{gathered}
C_{X, w}(\overline{\mathbb{R}}):=\operatorname{alg}_{\mathcal{M}_{X, w}(\mathbb{R})}(C(\overline{\mathbb{R}}) \cap V(\mathbb{R})), \quad P C_{X, w}:=\operatorname{alg}_{\mathcal{M}_{X, w}(\mathbb{R})}(P C \cap V(\mathbb{R})), \\
S O_{X, w}^{\diamond}:=\operatorname{alg}_{\mathcal{M}_{X, w}(\mathbb{R})}\left(\cup_{\lambda \in \mathbb{R}} S O_{\lambda}^{3}\right), \quad P S O_{X, w}^{\diamond}:=\operatorname{alg}_{\mathcal{M}_{X, w}(\mathbb{R})}\left(P C_{X, w} \cup S O_{X, w}^{\diamond}\right) .
\end{gathered}
$$

It is clear that $C_{X, w}(\overline{\mathbb{R}}) \subset C(\overline{\mathbb{R}}), P C_{X, w} \subset P C, S O_{X, w}^{\diamond} \subset S O^{\diamond}, P S O_{x, w}^{\diamond} \subset P S O^{\diamond}$, and all above algebras are contained in $P Q C$. Our last result is the following. Its proof will be given in Section 5.3

Theorem 2.9. Let $X(\mathbb{R})$ be either a separable rearrangement-invariant Banach function space with nontrivial Boyd indices or the variable Lebesgue space generated by an exponent $p \in \mathcal{B}_{M}(\mathbb{R})$. If one of the following conditions is fulfilled:

(i) $a \in P Q C$ and $b \in S O_{X, 1}^{\diamond}$;

(ii) $a \in S O^{\diamond}$ and $b \in P S O_{X, 1}^{\diamond}$;

(iii) $a \in \operatorname{alg}_{L^{\infty}(\mathbb{R})}(Q C \cup C(\overline{\mathbb{R}}))$ and $b \in \operatorname{alg}_{\mathcal{M}_{X, 1}(\mathbb{R})}\left(S O_{X, 1}^{\diamond} \cup C_{X, 1}(\overline{\mathbb{R}})\right)$;

then the commutator $a W^{0}(b)-W^{0}(b) a I$ is compact on the space $X(\mathbb{R})$.

For weighted Lebesgue spaces $L^{p}(\mathbb{R}, w)$ with $w \in A_{p}(\mathbb{R})$ a more general result was proved in [8, Theorem 4.4].

\section{Norm estimate for a Classical Calderón-Zygmund operator}

3.1. Pointwise estimate for the sharp maximal operator of $T f$ implies the norm estimate for $T$. For $\delta>0$ and $f \in L_{\text {loc }}^{\delta}(\mathbb{R})$, consider the sharp maximal operator defined by

$$
\left(M_{\delta}^{\#} f\right)(x):=\sup _{I \ni x} \inf _{c \in \mathbb{R}}\left(\frac{1}{|I|} \int_{I}|f(y)-c|^{\delta} d y\right)^{1 / \delta}
$$

where the supremum is taken over all finite intervals $I \subset \mathbb{R}$ containing $x$. If $\delta=1$, then this is the Fefferman-Stein sharp maximal operator.

The set of all bounded sublinear operators on the space $X(\mathbb{R}, w)$ will be denoted by $\mathcal{B}(X(\mathbb{R}, w))$. Obviously, $\mathcal{L}(X(\mathbb{R}, w)) \subset \mathcal{B}(X(\mathbb{R}, w))$. 
Theorem 3.1 ([15, Theorem 3.6]). Let $X(\mathbb{R})$ be a separable Banach function space and $w: \mathbb{R} \rightarrow[0, \infty]$ be a weight such that $w \in X_{\mathrm{loc}}(\mathbb{R})$ and $1 / w \in X_{\mathrm{loc}}^{\prime}(\mathbb{R})$. Suppose the Hardy-Littlewood maximal operator $M$ is bounded on $X(\mathbb{R}, w)$ and on $X^{\prime}\left(\mathbb{R}, w^{-1}\right)$. Assume that $0<\delta<1$ and $T: \mathfrak{M}(\mathbb{R}) \rightarrow \mathfrak{M}(\mathbb{R})$ is a linear operator such that

(a) $T$ is bounded on some $L^{p}(\mathbb{R})$ with $p \in(1, \infty)$;

(b) for every $f \in C_{0}^{\infty}(\mathbb{R})$, the operator $T$ satisfies the following pointwise estimate:

$$
\left(M_{\delta}^{\#}(T f)\right)(x) \leq C_{\delta}(M f)(x) \quad(x \in \mathbb{R})
$$

where $C_{\delta}$ is a positive constant depending only on $\delta$.

Then $T \in \mathcal{L}(X(\mathbb{R}, w))$ and

$$
\|T\|_{\mathcal{L}(X(\mathbb{R}, w))} \leq(1 / \lambda)^{\delta} C\|M\|_{\mathcal{B}(X(\mathbb{R}, w))}\|M\|_{\mathcal{B}\left(X^{\prime}\left(\mathbb{R}, w^{-1}\right)\right)} C_{\delta},
$$

where $\lambda \in(0,1)$ and $C>0$ are some absolute constants.

\subsection{Pointwise estimate for the sharp maximal operator of $T_{C Z} f$ with a classical Calderón-Zygmund operator $T_{C Z}$.}

Theorem 3.2 ([18, Lemma 2.2, Corollary 2.8]). Let $\delta \in(0,1)$ and $a \in C^{3}(\mathbb{R} \backslash\{0\})$ be such that

$$
B(a):=\max \left\{\left\|D_{0}^{j} a\right\|_{L^{\infty}(\mathbb{R})}: j=0,1,2,3\right\}<\infty,
$$

where the differential operator $D_{0}$ is defined by (2.2). Then

(a) the distribution $K=\mathcal{F}^{-1}$ a agrees with a function $K$ differentiable in $\mathbb{R} \backslash\{0\}$ and such that $|K(x)| \leq c_{0} B(a)|x|^{-1}$ and $\left|K^{\prime}(x)\right| \leq c_{1} B(a)|x|^{-2}$ for all $x \in \mathbb{R} \backslash\{0\}$, where the constants $c_{i} \in(0, \infty)$ depend only on $i=0,1$;

(b) for every $f \in C_{0}^{\infty}(\mathbb{R})$, the classical Calderón-Zygmund operator

$$
\left(T_{C Z} f\right)(x):=p . v . \int_{\mathbb{R}} K(x-y) f(y) d y \quad(x \in \mathbb{R})
$$

satisfies the following pointwise estimate:

$$
\left(M_{\delta}^{\#}\left(T_{C Z} f\right)\right)(x) \leq c(\delta) B(a)(M f)(x) \quad(x \in \mathbb{R})
$$

with

$$
c(\delta):=2^{2 / \delta-1}\left(2(1-\delta)^{-1 / \delta}\left(20+16 c_{1}\right)+32 c_{1}\right) .
$$

\subsection{Norm estimate.}

Theorem 3.3 ([18, Theorem 2.9]). Let $1<p<\infty$ and $w \in A_{p}(\mathbb{R})$. If a function $a \in C^{3}(\mathbb{R} \backslash\{0\})$ satisfies (3.2), then a classical Calderón-Zygmund operator $T_{C Z}$ given by (3.3) with the kernel $K=\mathcal{F}^{-1} a$ is bounded on the weighted Lebesgue space $L^{p}(\mathbb{R}, w)$ and

$$
\left\|T_{C Z}\right\|_{\mathcal{L}\left(L^{p}(\mathbb{R}, w)\right)} \leq c_{p, w} B(a),
$$

where $c_{p, w}$ is a positive constant depending only on $p$ and $w$.

Now we extend the previous result to the case of weighted Banach function spaces. 
Theorem 3.4. Let $X(\mathbb{R})$ be a separable Banach function space and $w: \mathbb{R} \rightarrow$ $[0, \infty]$ be a weight such that $w \in X_{\mathrm{loc}}(\mathbb{R})$ and $1 / w \in X_{\mathrm{loc}}^{\prime}(\mathbb{R})$. Suppose the HardyLittlewood maximal operator $M$ is bounded on $X(\mathbb{R}, w)$ and on $X^{\prime}\left(\mathbb{R}, w^{-1}\right)$. If a function $a \in C^{3}(\mathbb{R} \backslash\{0\})$ satisfies (3.2), then a classical Calderón-Zygmund operator $T_{C Z}$ given by (3.3) with the kernel $K=\mathcal{F}^{-1} a$ is bounded on the weighted Banach function space $X(\mathbb{R}, w)$ and

$$
\left\|T_{C Z}\right\|_{\mathcal{L}(X(\mathbb{R}, w))} \leq c_{X, w} B(a)
$$

where $c_{X, w}$ is a positive constant depending only on $X(\mathbb{R})$ and $w$.

Proof. In view of Theorem 3.3, the classical Calderón-Zygmund operator $T_{C Z}$ given by (3.3) is bounded on every Lebesgue space $L^{p}(\mathbb{R}), 1<p<\infty$, whence Condition (a) of Theorem 3.1 is fulfilled for $T=T_{C Z}$. Fix some $\delta \in(0,1)$. If $f \in C_{0}^{\infty}(\mathbb{R})$, then by Theorem 3.2,

$$
\left(M_{\delta}^{\#}\left(T_{C Z} f\right)\right)(x) \leq c(\delta) B(a)(M f)(x) \quad(x \in \mathbb{R}),
$$

where the constant $c(\delta)$ is given by (3.4). This means that Condition (b) of Theorem 3.1 also holds for $T=T_{C Z}$. Applying Theorem 3.1, we deduce that $T_{C Z} \in \mathcal{L}(X(\mathbb{R}, w))$ and (3.5) holds with

$$
c_{X, w}:=(1 / \lambda)^{\delta} C\|M\|_{\mathcal{B}(X(\mathbb{R}, w))}\|M\|_{\mathcal{B}\left(X^{\prime}\left(\mathbb{R}, w^{-1}\right)\right)} c(\delta)
$$

where $C>0$ and $\lambda \in(0,1)$ are some absolute constants.

\section{Proofs of the Boundedness Results}

4.1. Proof of Theorem 2.5. For $\lambda \in\{0, \infty\}$, Theorem 2.5 follows immediately from Theorem 3.4 because $W^{0}(a)$ is a classical Calderón-Zygmund operator with the kernel $K=\mathcal{F}^{-1} a$ and $B(a) \leq 6\|a\|_{S O_{\lambda}^{3}}$. Let $e_{\mu}(x):=e^{i \mu x}$ for all $\mu, x \in \mathbb{R}$. If $\lambda \in \mathbb{R} \backslash\{0\}$, then $a_{0}(x)=a(x+\lambda)$ belongs to $S O_{0}^{3}$ and $\left\|a_{0}\right\|_{S O_{0}^{3}}=\|a\|_{S O_{\lambda}^{3}}$. Moreover, $W^{0}(a)=e_{-\lambda} W^{0}\left(a_{0}\right) e_{\lambda} I$. Hence, by what has already been proved,

$$
\begin{aligned}
\left\|W^{0}(a)\right\|_{\mathcal{L}(X(\mathbb{R}, w))} & =\left\|e_{-\lambda} W^{0}\left(a_{0}\right) e_{\lambda} I\right\|_{\mathcal{L}(X(\mathbb{R}, w))}=\left\|W^{0}\left(a_{0}\right)\right\|_{\mathcal{L}(X(\mathbb{R}, w))} \\
& \leq c_{X, w}\left\|a_{0}\right\|_{S O_{0}^{3}}=c_{X, w}\|a\|_{S O_{\lambda}^{3}},
\end{aligned}
$$

which completes the proof.

4.2. The Boyd interpolation theorem for sublinear operators. The following theorem for linear operators was proved by Boyd [4, Theorem 1]. The same proof also works for sublinear operators because it relies on an estimate by Calderón [5, Theorem 8], which is also valid for sublinear operators. We also refer to [1, Theorem 5.16] and for the case of linear operators to [19, Theorem 2.b.11].

Theorem 4.1. Suppose $1<p<q<\infty$. If a sublinear operator is bounded on the Lebesgue spaces $L^{p}(\mathbb{R})$ and $L^{q}(\mathbb{R})$, then it is also bounded on a rearrangementinvariant space $X(\mathbb{R})$ with the Boyd indices satisfying

$$
1 / q<\alpha_{X} \leq \beta_{X}<1 / p
$$


4.3. Proof of Corollaries 2.2 and 2.6. Since $\alpha_{X}, \beta_{X} \in(0,1), w \in A_{1 / \alpha_{X}}(\mathbb{R})$ and $w \in A_{1 / \beta_{X}}(\mathbb{R})$, from [2, Theorem 2.31] it follows that, there are $p$ and $q$ such that

$$
1<q<1 / \beta_{X} \leq 1 / \alpha_{X}<p<\infty
$$

and $w \in A_{p}(\mathbb{R}) \cap A_{q}(\mathbb{R})$. Therefore $w \chi_{I} \in L^{p}(\mathbb{R}) \cap L^{q}(\mathbb{R})$ for every finite interval $I \subset \mathbb{R}$. By [19, Proposition 2.b.3], $w \chi_{I} \in X(\mathbb{R})$. From this fact and Axiom (A2) we obtain $w \chi_{E} \in X(\mathbb{R})$ for any measurable set $E$ of finite measure. Thus $w \in X_{\mathrm{loc}}(\mathbb{R})$. From Mukenhoupt's theorem [20, Theorem 2] we know that $M \in \mathcal{B}\left(L^{p}(\mathbb{R}, w)\right) \cap \mathcal{B}\left(L^{q}(\mathbb{R}, w)\right)$, whence the sublinear operator $A:=w M w^{-1} I$ is bounded on the Lebesgue spaces $L^{p}(\mathbb{R})$ and $L^{q}(\mathbb{R})$. By the Boyd interpolation theorem (Theorem 4.1), the operator $A$ is bounded on the rearrangement-invariant space $X(\mathbb{R})$, whence the Hardy-Littlewood operator $M$ is bounded on the space $X(\mathbb{R}, w)$. From $\alpha_{X}, \beta_{X} \in(0,1)$ and [1, Chap. 3, Proposition 5.13] we deduce that $\alpha_{X^{\prime}}=1-\beta_{X}, \beta_{X^{\prime}}=1-\alpha_{X} \in(0,1)$ and $1 / w \in A_{1 / \alpha_{X^{\prime}}}(\mathbb{R}) \cap A_{1 / \beta_{X^{\prime}}}(\mathbb{R})$. As before we can show that $1 / w \in X_{\mathrm{loc}}^{\prime}(\mathbb{R})$ and that the operator $B:=w^{-1} M w I$ is bounded on the associate space $X^{\prime}(\mathbb{R})$. Therefore, the operator $M$ is bounded on the space $X^{\prime}\left(\mathbb{R}, w^{-1}\right)$. To complete the proof of Corollary 2.2 (resp. Corollary 2.6), it remains to apply Theorem 2.1 (resp. Theorem 2.5).

4.4. Proof of Corollaries 2.3 and 2.7. Since $p_{+}<\infty$, from [7, Theorem 2.78] we deduce that the space $L^{p(\cdot)}(\mathbb{R})$ is separable. If $p$ satisfies $(1.1)$, then it is easy to see that $p \in L H(\mathbb{R})$ if and only if $p^{\prime} \in L H(\mathbb{R})$. From [7, Theorem 2.80, Corollary 2.81] it follows that the associate space to $L^{p(\cdot)}(\mathbb{R})$ is isomorphic to $L^{p^{\prime}(\cdot)}(\mathbb{R})$. Hence $w \in A_{L^{p(\cdot)}}(\mathbb{R})$ is equivalent to

$$
\sup _{I} \frac{1}{|I|}\left\|w \chi_{I}\right\|_{L^{p \cdot(\cdot)}(\mathbb{R})}\left\|w^{-1} \chi_{I}\right\|_{L^{p^{\prime}(\cdot)(\mathbb{R})}}<\infty
$$

where the supremum is taken over all finite intervals $I \subset \mathbb{R}$. From (4.1) we immediately get $w \chi_{I} \in L^{p(\cdot)}(\mathbb{R})$ and $w^{-1} \chi_{I} \in L^{p^{(} \cdot(\cdot)}(\mathbb{R})$ for any finite interval $I$. By Axiom (A2), this implies that $w \chi_{E} \in L^{p(\cdot)}(\mathbb{R})$ and $w^{-1} \chi_{E} \in L^{p^{\prime}(\cdot)}(\mathbb{R})$ for any measurable set $E \subset \mathbb{R}$ of finite measure. Hence $w$ belongs to $L_{\text {loc }}^{p(\cdot)}(\mathbb{R})$ and $1 / w$ lies in $L_{\text {loc }}^{p^{\prime}(\cdot)}(\mathbb{R})$. By [6, Theorem 1.5], the operator $M$ is bounded on the weighted variable Lebesgue space $L^{p(\cdot)}(\mathbb{R}, w)$ if and only if $w$ satisfies (4.1). From (4.1) we see that $w \in A_{L^{p(\cdot)}}(\mathbb{R})$ if and only if $1 / w \in A_{L^{p^{(\cdot)}}}(\mathbb{R})$. Thus, if $w \in A_{L^{p(\cdot)}}(\mathbb{R})$, then $M$ is bounded on both $L^{p(\cdot)}(\mathbb{R}, w)$ and $L^{p^{\prime}(\cdot)}\left(\mathbb{R}, w^{-1}\right)$. The latter space is isomorphic to the associate space $\left[L^{p(\cdot)}(\mathbb{R}, w)\right]^{\prime}$ in view of $[15$, Lemma $2.4(\mathrm{c})]$ and [7, Theorem 2.80, Corollary 2.81]. To complete the proof of Corollary 2.3 (resp. Corollary 2.7), it remains to apply Theorem 2.1 (resp. Theorem 2.5).

4.5. Proof of Corollaries 2.4 and 2.8. Since $1<p_{-}, p_{+}<\infty$ for $p \in \mathcal{B}_{M}(\mathbb{R})$, from [7, Theorems 2.78, 2.80, Corollary 2.81] we know that $L^{p(\cdot)}(\mathbb{R})$ is separable and that its associate space is isomorphic to $L^{p^{\prime}(\cdot)}(\mathbb{R})$. By the hypothesis, the Hardy-Littlewood maximal operator $M$ is bounded on $L^{p(\cdot)}(\mathbb{R})$. In view of [9, Theorem 5.7.2], the latter is equivalent to the boundedness of $M$ on the space $L^{p^{\prime}(\cdot)}(\mathbb{R})$. To complete the proof of Corollary 2.4 (resp. Corollary 2.8), it remains to apply Theorem 2.1 (resp. Theorem 2.5). 


\section{Proof of the COMPACTNESS ReSUlT}

5.1. Compactness of commutators on weighted Lebesgue spaces. The following theorem is one of the main ingredients of the proof of Theorem 2.9.

Theorem 5.1. Let $1<p<\infty$ and $w \in A_{p}(\mathbb{R})$. If one of the following conditions is fulfilled:

(i) $a \in P Q C$ and $b \in S O_{L^{p}, w}^{\diamond}$;

(ii) $a \in S O^{\diamond}$ and $b \in P S O_{L^{p}, w}^{\diamond}$;

(iii) $a \in \operatorname{alg}_{L^{\infty}(\mathbb{R})}(Q C \cup C(\overline{\mathbb{R}}))$ and $b \in \operatorname{alg}_{\mathcal{M}_{L^{p}, w}(\mathbb{R})}\left(S O_{L^{p}, w}^{\diamond} \cup C_{L^{p}, w}(\overline{\mathbb{R}})\right)$;

then the commutator $a W^{0}(b)-W^{0}(b) a I$ is compact on the weighted Lebesgue space $L^{p}(\mathbb{R}, w)$.

A more general result was obtained in [8, Theorem 4.4] (see also [17, Theorem 4.6]).

5.2. Transferring the compactness from standard Lebesgue spaces. The following result was proved in [14, Corollary 2.3] in the case of rearrangement invariant spaces over finite measure spaces, however, the proof is the same in the case of $X(\mathbb{R})$.

Lemma 5.2. Suppose a linear operator $A$ is bounded on all Lebesgue spaces $L^{p}(\mathbb{R})$ with $p \in(1, \infty)$. Then $A$ is compact on a rearrangement-invariant space $X(\mathbb{R})$ with nontrivial Boyd indices if and only if it is compact on a Lebesgue space $L^{r}(\mathbb{R})$ with some $r \in(1, \infty)$.

The following result obtained in [12, Lemma 6.4] is an analogue of Lemma 5.2 for variable Lebesgue spaces.

Lemma 5.3. Suppose a linear operator $A$ is bounded on all variable Lebesgue spaces $L^{p(\cdot)}(\mathbb{R})$ with $p \in \mathcal{B}_{M}(\mathbb{R})$. If $A$ is compact on a Lebesgue space $L^{r}(\mathbb{R})$ with $r \in(1, \infty)$, then $A$ is compact on all $L^{p(\cdot)}(\mathbb{R})$ with $p \in \mathcal{B}_{M}(\mathbb{R})$.

5.3. Proof of Theorem 2.9. From Corollaries 2.2, 2.6 and 2.4, 2.8 it follows that the algebras $C_{X, 1}(\overline{\mathbb{R}}), P C_{X, 1}, S O_{X, 1}^{\diamond}$, and $P S O_{X, 1}^{\diamond}$ are well defined. From their definitions we conclude that there is a sequence $b_{n} \in \mathcal{M}_{X, 1}(\mathbb{R})$ such that

$\left(\mathrm{i}^{\prime}\right) b_{n} \in\left(S O^{3}\right)^{\diamond},\left(\mathrm{ii}^{\prime}\right) b_{n} \in\left(S O^{3}\right)^{\diamond} \cup(P C \cap V(\mathbb{R})),\left(\mathrm{iii}^{\prime}\right) b_{n} \in\left(S O^{3}\right)^{\diamond} \cup(C(\overline{\mathbb{R}}) \cap V(\mathbb{R}))$, in cases (i)-(iii), respectively, where $\left(S O^{3}\right)^{\diamond}:=\cup_{\lambda \in \mathbb{R}} S O_{\lambda}^{3}$, and

$$
\left\|W^{0}\left(b_{n}\right)-W^{0}(b)\right\|_{\mathcal{L}(X(\mathbb{R}))}=\left\|b_{n}-b\right\|_{\mathcal{M}_{X, 1}(\mathbb{R})} \rightarrow 0 \quad \text { as } \quad n \rightarrow \infty .
$$

In each case (i)-(iii), the operators $a W^{0}\left(b_{n}\right)-W^{0}\left(b_{n}\right) a I$ are compact for all $n \in \mathbb{N}$ on every $L^{p}(\mathbb{R}), 1<p<\infty$, in view of Theorem 5.1. From Lemmas 5.2-5.3 we deduce that the operators $a W^{0}\left(b_{n}\right)-W^{0}\left(b_{n}\right) a I$ are compact for all $n \in \mathbb{N}$ on the space $X(\mathbb{R})$. This observation and (5.1) yield the compactness of the commutator $a W^{0}(b)-W^{0}(b) a I$ on the space $X(\mathbb{R})$ in each case (i)-(iii).

Acknowledgement. This work was partially supported by the Fundação para a Ciência e a Tecnologia (Portuguese Foundation for Science and Technology) 
through the projects UID/MAT/00297/2013 (Centro de Matemática e Aplicações) and EXPL/MAT-CAL/0840/2013 (Problemas Variacionais em Espaços de Sobolev de Expoente Variável).

\section{REFERENCES}

1. C. Bennett and R. Sharpley, Interpolation of Operators. Academic Press, New York, NY, 1988.

2. A. Böttcher and Yu.I. Karlovich, Carleson Curves, Muckenhoupt weights, and Toeplitz Operators. Progress in Mathematics 154. Basel, Birkhäuser, 1997.

3. A. Böttcher, Yu.I. Karlovich, and I.M. Spitkovsky, Convolution Operators and Factorization of Almost Periodic Matrix Functions. Birkhäuser, Basel, 2002.

4. D.W. Boyd, Spaces between a pair of reflexive Lebesgue spaces. Proc. Amer. Math. Soc. 18 (1967), 215-219.

5. A.P. Calderón, Spaces between $L^{1}$ and $L^{\infty}$ and the theorem of Marcinkiewicz. Studia Math. 26 (1966), 273-299.

6. D. Cruz-Uribe, A. Fiorenza, and C.J. Neugebauer, Weighted norm inequalities for the maximal operator on variable Lebesgue spaces. J. Math. Anal. Appl. 394 (2012), 744-760.

7. D. Cruz-Uribe and A. Fiorenza, Variable Lebesgue Spaces. Birkhäuser, Basel, 2013.

8. I. De la Cruz-Rodríguez, Yu.I. Karlovich, and I. Loreto-Hernández, Commutators of convolution type operators with piecewise quasicontinuous data. Commun. Math. Anal. 17 (2014), no. 2, 131-150.

9. L. Diening, P. Harjulehto, P. Hästö, and M. Růžička, Lebesgue and Sobolev Spaces with Variable Exponents. Lecture Notes in Mathematics 2017, Springer, Berlin, 2011.

10. R. Duduchava, Integral Equations with Fixed Singularities. Teubner Verlagsgesellschaft, Leipzig, 1979.

11. R. Hunt, B. Muckenhoupt and R. Wheeden, Weighted norm inequalities for the conjugate function and Hilbert transform. Trans. Amer. Math. Soc. 176 (1973), 227-251.

12. A.Yu. Karlovich, Maximally modulated singular integral operators and their applications to pseudodifferential operators on Banach function spaces. In Function Spaces VII. Contemp. Math. (to appear), http://arxiv.org/abs/1408.4400.

13. A.Yu. Karlovich, Banach algebra of the Fourier multipliers on weighted Banach function spaces. Concr. Oper. 2 (2015), 27-36.

14. A.Yu. Karlovich and Yu.I. Karlovich, Compactness of commutators arising in the Fredholm theory of singular integral operators with shifts. In "Factorization, singular operators and related problems. Proceedings of the conference in honor of Professor Georgii Litvinchuk, Funchal, Madeira, Portugal, January 28-February 1, 2002." Kluwer Academic Publishers, Dordrecht, 2003, 111-129.

15. A.Yu. Karlovich and I.M. Spitkovsky, The Cauchy singular integral operator on weighted variable Lebesgue spaces. In "Concrete Operators, Spectral Theory, Operators in Harmonic Analysis and Approximation", Birkhäuser, Basel. Operator Theory: Advances and Applications 236 (2014), 275-291.

16. Yu.I. Karlovich and I. Loreto Hernández, Algebras of convolution type operators with piecewise slowly oscillating data. I: Local and structural study. II: Local spectra and Fredholmness. Part I: Integral Equations Operator Theory 74 (2012), 377-415; Part II: Integral Equations Operator Theory 75 (2013), 49-86.

17. Yu.I. Karlovich and I. Loreto Hernández, On convolution type operators with piecewise slowly oscillating data. In "Operator theory, pseudo-differential equations, and mathematical physics. The Vladimir Rabinovich anniversary volume", Birkhäuser, Basel. Operator Theory: Advances and Applications 228 (2013), 185-207.

18. Yu.I. Karlovich and J. Loreto Hernández, Wiener-Hopf operators with slowly oscillating matrix symbols on weighted Lebesgue spaces. Integral Equations Operator Theory 64 (2009), 203-237. 
19. J. Lindenstrauss and L. Tzafriri, Classical Banach Spaces. II: Function Spaces. Springer, Berlin, 1979.

20. B. Muckenhoupt, Weighted norm inequalities for the Hardy maximal function. Trans. Amer. Math. Soc. 165 (1972), 207-226.

21. D. Sarason, Functions of vanishing mean oscillation. Trans. Amer. Math. Soc. 207 (1975), 391-405.

22. D. Sarason, Toeplitz operators with piecewise quasicontinuous symbols. Indiana Univ. Math. J. 26 (1977), 817-838.

Centro de Matemática e Aplicações (CMA) and Departamento de Matemática, Faculdade de Ciências e Tecnologia, Universidade Nova de lisboa, Quinta da Torre, 2829-516 Caparica, Portugal.

E-mail address: oyk@fct.unl.pt 